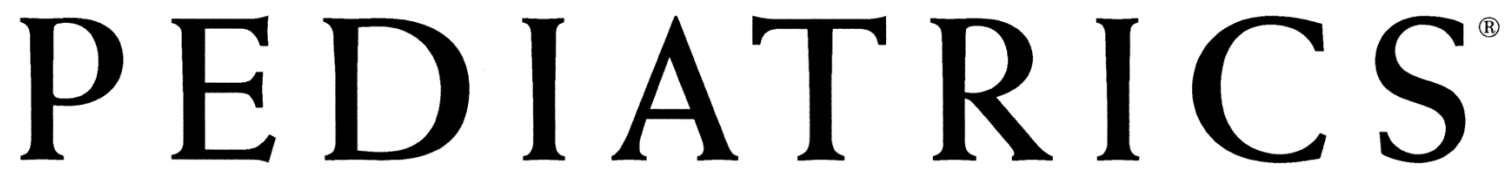

OFFICIAL JOURNAL OF THE AMERICAN ACADEMY OF PEDIATRICS

\title{
Effect of Botulinum Toxin in the Treatment of Drooling: A Controlled Clinical
}

\section{Trial}

Peter H. Jongerius, Frank J.A. van den Hoogen, Jacques van Limbeek, Fons J.

Gabreëls, Karen van Hulst and Jan J. Rotteveel

Pediatrics 2004;114;620

DOI: $10.1542 /$ peds.2003-1104-L

The online version of this article, along with updated information and services, is

located on the World Wide Web at:

http://pediatrics.aappublications.org/content/114/3/620.full.html

PEDIATRICS is the official journal of the American Academy of Pediatrics. A monthly publication, it has been published continuously since 1948. PEDIATRICS is owned, published, and trademarked by the American Academy of Pediatrics, 141 Northwest Point Boulevard, Elk Grove Village, Illinois, 60007. Copyright (c) 2004 by the American Academy of Pediatrics. All rights reserved. Print ISSN: 0031-4005. Online ISSN: 1098-4275.

\section{American Academy of Pediatrics}

DEDICATED TO THE HEALTH OF ALL CHILDREN ${ }^{\mathrm{m}}$ 


\title{
Effect of Botulinum Toxin in the Treatment of Drooling: A Controlled Clinical Trial
}

\author{
Peter H. Jongerius, MD*; Frank J.A. van den Hoogen, MD, PhD ; Jacques van Limbeek, MD, PhD§; \\ Fons J. Gabreëls, MD, PhD*; Karen van Hulst, BSc\|; and Jan J. Rotteveel, MD, PhD\|
}

\begin{abstract}
Objective. To investigate the clinical effectiveness of botulinum neurotoxin type A (BoNT) to reduce drooling in children with cerebral palsy $(\mathrm{CP})$.

Methods. A controlled clinical trial was performed in which the results of single-dose BoNT injections in the submandibular glands were compared with treatment with scopolamine. Forty-five children who had CP and experienced severe drooling were enrolled. Drooling severity was measured at baseline, during application of scopolamine, and at different intervals after BoNT injections up to 24 weeks, using the Drooling Quotient (DQ), the Teacher Drooling Scale (TDS), and Visual Analog Scales (VAS).
\end{abstract}

Results. Drooling was reduced during scopolamine application as well as after BoNT injections. Compared with baseline, the mean DQ showed a significant decrease throughout the study. Greatest reductions were achieved 2 to 8 weeks after BoNT injection. No significant differences were found between scopolamine measurements and those up to 24 months after BoNT injection. Using VAS, parents recorded the effect on drooling in which significant differences were found between baseline VAS score and all follow-up assessments. According to our definition of "success to therapy," demanding a 2-point decrease on the TDS, $61.5 \%$ of patients responded to BoNT injections. Analysis of the DQ demonstrated a response rate of $53 \%$ of the patients to scopolamine and $48.7 \%$ to BoNT until 24 weeks after BoNT injections, the actual duration of this study. As a reaction to scopolamine, $71.1 \%$ of the patients had moderate to severe side effects. Only nonsevere, incidental side effects were reported after BoNT injections.

Conclusions. During scopolamine application as well as after intraglandular BoNT injections, a clinically relevant reduction in drooling was achieved in children with $\mathrm{CP}$, demonstrating maximum effect 2 to 8 weeks after injections. This is the first controlled clinical trial that confirmed a significant effect of BoNT injections in the treatment of drooling. General anesthesia was needed for all children. BoNT injections show fewer and less serious side effects than transdermal scopolamine treatment. $\mathrm{Pe}$ -

From the *Department of Rehabilitation, University Medical Centre St Radboud, Nijmegen, Netherlands; 抽epartment of Otorhinolaryngology, University Medical Centre St Radboud, Nijmegen, Netherlands; §Sint Maartenskliniek, Rehabilitation Centre, Nijmegen, Netherlands; and ||Department of Pediatric Neurology, University Medical Centre St Radboud, Nijmegen, Netherlands.

Accepted for publication Mar 15, 2004.

DOI: 10.1542/peds.2003-1104-L

Reprint requests to (P.H.J.) Department of Rehabilitation, University Medical Centre St Radboud, PO Box 9101 6500HB, Nijmegen 720, Netherlands. E-mail: p.jongerius@reval.umcn.nl

PEDIATRICS (ISSN 0031 4005). Copyright (C) 2004 by the American Academy of Pediatrics. diatrics 2004;114:620-627; salivary flow, drooling, cerebral palsy, botulinum toxin, child.

ABBREVIATIONS. CP, cerebral palsy; DQ, Drooling Quotient; TDS, Teacher Drooling Scale; BoNT, botulinum neurotoxin type A; VAS, Visual Analog Scales; WCS, worst-case scenario; MANOVA, multivariate analysis of variance.

$\mathrm{D}$ rooling is an important clinical problem in $\sim 10 \%$ to $38 \%$ of patients with cerebral palsy (CP). ${ }^{1-3}$ Insufficient control of the coordinate mechanism of orofacial, palatolingual, and head and neck musculature results in excessive pooling of saliva in the anterior part of the oral cavity and unintentional saliva loss. ${ }^{4}$ Hypersalivation is generally not the case in children with CP. Direct saliva collection can be performed by the swab method in which absorbent cotton rolls are placed directly at the orifices of the glands for 5-minutes. The flow rate can be calculated by the following formula: salivary flow rate $(\mathrm{mg} / \mathrm{min})=($ weight increase of rolls $[\mathrm{mg}] /$ time of collection [min]).5,6

The clinical evaluation of drooling severity and frequency is difficult because of within-subject fluctuation during the day and a large between-subject variation. Several systems have been used and advocated for assessment of the extent of drooling. Since its introduction, various modifications of the Drooling Quotient (DQ) have been used. ${ }^{7-13}$ The DQ is a validated, semiquantitative, direct observational method (see Methods). ${ }^{7,14}$ Rating scales such as the Teacher Drooling Scale (TDS) have been designed to assess drooling severity and frequency. The TDS is a useful tool for outpatient visits. ${ }^{15}$

The management of drooling remains a problem. Despite effective treatment modalities to diminish saliva production, drooling may persist. Many factors contribute to the saliva passage from the oral cavity to the esophagus, such as the child's mental abilities, the cognitive awareness of social norms, an intact swallowing mechanism, oral sensibility, lip closure, and the ability to hold the head in an upright position. In addition, variables that may influence salivary flow rate are medication, circadian rhythms, prestimulation, gender, age, psychological effect, and general health. ${ }^{16-21}$ The effective reduction of saliva production is relevant to the patient only when the treatment leads to a clinically apparent diminished drooling.

Conservative treatments as well as surgical proce- 
dures all have their limitations. ${ }^{22}$ Recent reports suggested botulinum neurotoxin type A (BoNT) injections into the salivary glands as an option for treatment of drooling. $6,9,10$ These reports are case series and small cohort studies that lack the power to prove the efficacy of BoNT injections. Ellies et $\mathrm{al}^{23}$ studied a larger population and concluded that the BoNT effect lasted for $\sim 2$ to 3 months. From the pharmacologic profile of BoNT, an appreciable anticholinergic effect can be expected. In particular, type A toxin cleaves SNAP-25, an enzyme involved in the release of acetylcholine at the presynaptic membrane of parasympathetic nerves. In this way, a temporary denervation of the target organ is established. Botulinum toxin is known to give clinically relevant results for 9 to 12 months in other conditions involving the autonomic nervous system, for example hyperhidrosis. ${ }^{24,25}$ Although BoNT has been suggested for clinical use in the treatment of drooling, uncertainty remains about the clinical effect and duration.

In this study, a controlled clinical trial on the treatment of drooling was performed in which BoNT injections in the submandibular glands were compared with scopolamine treatment. Difference scores of semiquantitative measurements of drooling (DQ, TDS, Visual Analog Scales [VAS]) were analyzed. The null hypothesis that the effect on drooling would not differ between scopolamine and BoNT was tested.

\section{METHODS}

Forty-five children with the diagnosis $\mathrm{CP}$ were recruited from the outpatient clinic and enrolled in the study between January 2000 and November 2001. Consecutive patients were included during a qualification period in which inclusion criteria and exclusion criteria were examined (Table 1).

A score of 3 or higher on the TDS (Table 2) was mandatory to be included in the study. ${ }^{15}$ All drugs used were evaluated carefully to assess their influence on saliva secretion. Drugs to treat drooling had to be stopped at least 3 months before participation. Throughout the study, no medication that could influence the severity of drooling was allowed. No requirements were set with regard to the child's level of mental development.

Possible adverse effects and risks related to the interventions

\section{TABLE 1. Inclusion and Exclusion Criteria}

\begin{tabular}{l} 
Inclusion criteria \\
Children (male and female) of preschool and school age \\
(subjects aged $3-18$ y) \\
Diagnosis of CP \\
A score of $\geq 3$ on the TDS, indicating severe drooling 38 \\
All medication, taken to treat drooling, stopped at least 3 mo \\
before start of the study \\
Minimal body weight $8 \mathrm{~kg}$ \\
Informed consent obtained \\
Caregivers have high enough cognitive ability to participate \\
in the study \\
Readiness to participate for $\sim 8$ mo \\
Exclusion criteria \\
Child is enrolled in another medical study \\
Previous surgical procedures in the oral/nasal cavity \\
interfering with saliva production \\
Treatment with BoNT for another indication in the previous 6 \\
mo \\
Known hypersensitivity to Botox or any part of the \\
formulation \\
Use of drugs that interfere with saliva secretion \\
Known systemic diseases (bronchial asthma, congenital heart \\
failure, and myasthenia gravis) \\
\hline
\end{tabular}

TABLE $2 . \quad$ TDS

$\begin{array}{ll}1 & \text { No drooling } \\ 2 & \text { Infrequent drooling; small amount } \\ 3 & \text { Occasional drooling; intermittent all day } \\ 4 & \text { Frequent drooling but not profuse } \\ 5 & \text { Constant drooling; always wet }\end{array}$

during the study were explained to the parents. Written informed consent was obtained. The Hospital's Human Research Committee approved the study.

\section{Study Design}

The study was executed as a controlled, open-label, clinical trial. The difference between the 2 episodes of treatment had to be at least $1 \mathrm{SD}$. Drooling evaluation (see Procedures) was performed during baseline, scopolamine application, and after BoNT injections into the submandibular glands. The sequence of interventions had a fixed order: scopolamine before BoNT. This was chosen because the washout period of scopolamine is known, whereas the duration of the supposed effect of BoNT needed to be determined. An independent employee assessed the primary outcome parameters blinded for the status of the participating patients, which was achieved by varying the number of baseline measurements among the patients according to a predetermined schedule.

In view of side effects of scopolamine, the protocol anticipated that some of the patients might not complete the scopolamine period. When scopolamine was used for $<48$ hours, the patient was considerate a dropout. When scopolamine was discontinued $>48$ hours after start, the patient remained in the study, provided that a control measurement was obtained within the first 24 hours after discontinuation of the therapy.

Patients returned for follow-up measurements at 2, 4, 8, 16, and 24 weeks after BoNT injections. Patients had to have undergone at least 1 of the scheduled investigations at 2, 4, or 8 weeks. At least 3 of 5 visits within the first 24 weeks after BoNT injections had to be conducted.

If a patient was excluded during the use of scopolamine or during the washout period, it was planned to contact the parents by telephone after 2 and 4 weeks to check for adverse effects or other complaints. Dropouts after the BoNT injections were to be contacted monthly until 24 weeks after injection.

\section{Procedures}

A scopolamine patch (Scopo-derm TTS; Novartis Consumer Health BV, Breda, Netherlands) was placed behind the ear and changed within every 72 hours. An assessment was scheduled on the 10th day, with the 4th plaster in situ being applied no longer than 48 hours before. After a washout period of 2 to 4 weeks, the child was admitted for outpatient treatment. Using general anesthesia for all patients, a single dose of botulinum toxin (Botox; Allergan, Nieuwegein, Netherlands), reconstituted with $0.9 \%$ sodium chloride solution, was injected bilaterally in the submandibular glands using a 25-G needle (Spinocan) and a 1-mL syringe. Weight-dependent dosages were injected in each gland: 15 $\mathrm{U} /$ gland for children who weighed $<15 \mathrm{~kg}, 20 \mathrm{U} /$ gland for children with a body weight of between $15 \mathrm{~kg}$ and $25 \mathrm{~kg}$, and 25 $\mathrm{U} /$ gland for children who weighed $>25 \mathrm{~kg}$. On injection, each dose was fractionated and divided over minimally 3 sites in the gland. Ultrasound guidance was performed using a system (SAL 250; Toshiba Medical Systems Corporation, Tokyo, Japan) equipped with a $7.5-\mathrm{MHz}$ transducer. Only the submandibular glands were injected. It is generally accepted that these glands produce $60 \%$ to $70 \%$ of secreted resting saliva when the individual is not eating or drinking. ${ }^{26}$ Saliva that is produced during eating and drinking is produced mainly by the parotid glands, with which we did not want to interfere. The sublingual glands, contributing up to $5 \%$ to the total saliva production, were not treated.

After BoNT, parents were asked to register all possible side effects in a diary. These were discussed during outpatient visits.

\section{Assessment of Drooling}

The DQ and VAS served as the primary outcome measures for this study. The TDS, scored on an ordinal scale, was used to give supportive evidence for the efficacy of BoNT. 
The DQ was scored according to its original design during 2 periods of 10 minutes separated by a 60 -minute break. ${ }^{7}$ An episode of drooling was defined as new saliva present on the lip margin or dropping from the chin. ${ }^{12}$ Every 15 seconds (40 observations in 10 minutes), the presence or absence of drooling was assessed. Patients were evaluated at least 1 hour after a meal while awake and sitting erect. Two speech therapists were especially trained to execute the measurements. Separate observations were made during different activities: 1 with the child watching television and 1 during an activity, as chosen by the child, that demanded a higher level of concentration or physical effort. The mean of the 2 observations was used for analysis to provide an outcome on a numeric scale.

The DQ was expressed as a percentage estimated from the ratio of observed drooling episodes and the total number of observations $(\mathrm{DQ}[\%]=100 \times$ number of drooling episodes $/ 40) .{ }^{8} \mathrm{DQ}$ assessments were made at baseline, during the use of scopolamine, at washout after ending scopolamine therapy, and at regular intervals after BoNT injections (2, 4, 8, 16, and 24 weeks).

After receiving specific instructions, parents filled out VAS to investigate therapy results as experienced in the home situation. Scales of exactly $10 \mathrm{~cm}$ without visible subdivisions were presented on which the average degree of drooling severity during the 10 to 14 days before assessment had to be indicated. A mark at the left end represented severe drooling; a mark at the right end meant no drooling. An independent employee scored the VAS with a ruler in millimeters, resulting in a number ranging from 0 to 100 , which was handled as a parametric variable. VAS assessment was made at baseline, during the use of scopolamine, and at regular intervals after BoNT injections $(4,8,16$, and 24 weeks).

The TDS (Table 2) was used to assess the degree of drooling by interviewing the parents or caregivers during outpatient visits. ${ }^{15}$ Assessments of TDS were made at baseline and after BoNT injections ( 8 and 24 weeks). Before analysis of the data, a significant reaction to therapy was defined as a 2-point improvement on the TDS (range: 1-5; Table 2). The outcome after BoNT injections is used only to support the findings in the DQ and VAS data.

\section{Statistics}

A power analysis was performed before start of the study. Approximately 40 patients were needed to reach a power of $80 \%$ with an $\alpha$ of .05 . The difference between the 2 episodes of treatment had to be at least $1 \mathrm{SD}$. Assuming a dropout percentage of $7 \%$, the inclusion of 45 patients was sufficient. Because of the complexity of the design and vulnerability of the patients, missing data were inevitable. For this reason, data were adjusted in 2 ways: 1) by carrying the last observation forward and 2) by a worst-case scenario (WCS) system. In the WCS procedure, all missing data were replaced by baseline values. In this way, the effect difference between the therapies was "reduced" by intro- ducing a bias toward the null. The outcomes of both approaches were compared.

All statistical procedures were conducted with SPSS / pc+ (version 9.0; SPSS, Inc, Chicago, IL). Data analysis included descriptive statistics; multivariate analysis of variance (MANOVA) of repeated measurements to identify patterns of response over time, using a within-subjects design with the measurement moments as the variables; and paired-samples $t$ tests to analyze differences of paired observations (DQ and VAS) at subsequent measurements. In addition, success of therapy for either scopolamine or BoNT was defined as a decrease in DQ of at least $50 \%$ of the patient's baseline value. Frequency analyses were performed to determine the percentage of responders in the population. A Wilcoxon signed ranks test was used to analyze changes in TDS (ordinal scale). For all statistics, a level of significance with a 1-sided $P \leq$ .05 was mandatory.

\section{RESULTS}

Forty-five children were included: 28 boys and 17 girls (age: 3-17 years; mean: 9.5; SD: 3.7). Eight were ambulant without aid, 37 had wheelchairs, 22 could not talk, 29 attended a special education school, and 14 went to a child care center for mentally handicapped children. Thirty-four children had mental retardation with a developmental level $<6$ years as determined by psychological investigation.

None of the patients was treated with botulinum toxin before. By the end of the trial, 6 dropouts had occurred: 4 patients could not fulfill the scopolamine period, 1 changed antiepileptic medication, and 1 did not attend the required measurements because of an intercurrent illness not related to the trial. The results of 39 patients could be analyzed.

\section{DQ}

In the first instance, analyses were executed with carrying the last observation forward data. Descriptive statistics of the consecutive measurement moments showed that, compared with baseline, the mean DQ decreased during scopolamine application as well as after BoNT injections, which is shown in Fig 1. With the DQ at each moment in time defined as the within-subjects variable, a MANOVA with a repeated measurements design was executed to eval-
Fig 1. DQ: mean in time. Sc indicates application of Scopolamine; BTX, injection of botulinum toxin.

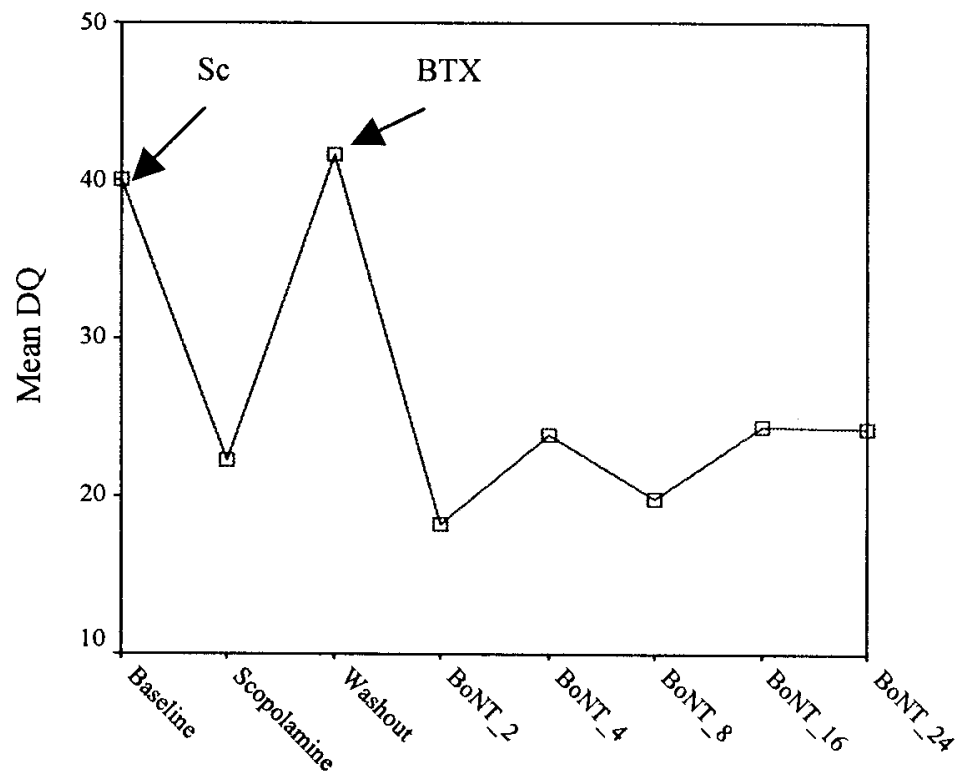


uate the pattern of response during the trial (Hotelings trace: $F=12.79 ; d f$ [7.00]; $P=.000$ ). An effect over time was found as depicted in Fig 1 and described in additional detail by analysis of difference.

Univariate analyses were executed to analyze further interval difference scores. To justify the length of the washout period, we compared the DQ values at baseline with the measurements after washout of scopolamine, using a paired-samples $t$ test. The mean change in DQ from baseline to washout value was estimated to be -1.60 , which was a nonsignificant difference ( $t=-0464 ; d f$ [38]; $P=.32,1$ tailed). This finding minimizes the probability of a carryover effect from scopolamine treatment to BoNT, indicating that the length of washout after scopolamine application was sufficient.

Changes in DQ (expressed as differences), SD, and $P$ values between baseline and the subsequent measurements are tabulated in Table 3. All DQ measurements showed a significant decrease (mean differences: 15.5-21.7; $P \leq .05$, based on paired samples $t$ tests).

The greatest reduction was achieved 2 weeks after BoNT injections (BoNT-2: mean difference score: 21.7; SD: $18.3 ; P=.000)$. The DQ increased slightly after BoNT-2. At the end of the study, though, a significant reduction of drooling was still found, suggesting an ongoing effect.

During additional analysis, scopolamine measurements were compared with the measurements after BoNT injections up to 24 weeks (Table 4). No significant differences were found (mean differences: -2.2 to $4.1 ; P>.05$, based on paired samples $t$ tests). Analysis of WCS data did not lead to different results comparing baseline with both treatments.

According to our definition of success to therapy, patients could be assigned as a responder when baseline DQ decreased by $50 \%$ or more during the interventions. Analysis of frequencies was done. During scopolamine, $53 \%$ of the patients were recognized as responders. Response rates to BoNT were as follows: BoNT-2, 64.1\%; BoNT-4, 43.5\%; BoNT-8, 53\%; BoNT16, 41\%; and BoNT-24, $48.7 \%$.

\section{VAS}

The analysis of the VAS showed an effect over time. With the VAS at each moment in time defined as the within-subjects variable, a MANOVA with a repeated measurements design was executed to evaluate the pattern of response during the trial (Hotelings trace: $F=16.55 ; d f[5] ; P=.000)$. Figure 2 shows the course of the mean scores at the subsequent measurement moments.

The degree of reported drooling by the parents declined substantially when baseline numbers (VAS: 19.3; SD: 13.4; range: 2-61) were compared with measurement during scopolamine application (VAS: 53.6; SD: 13.4; range: 0-96). Measurements scored by the parents after BoNT demonstrated good results with a maximum reduction at BoNT-4 (VAS: 49.3; SD: 24.5; range: 8-93). The VAS score at BoNT-4 was in the same range as during scopolamine application, revealing no significant difference (mean difference: 4.2; SD: 31.3; $t-0842 ; d f=38 ; P=.20,1$ tailed). Analyses of VAS difference scores were performed, using paired-samples $t$ tests to compare baseline values with the results after therapy (Table 3 ). All differences were significant, indicating that parents reported diminished drooling throughout the study. In addition, scopolamine VAS scores were compared with all subsequent BoNT measurements (Table 4), using paired-samples $t$ tests. No differences were found, meaning that the outcomes of both therapies as reported by the parents were in the same range.

\section{TDS}

Patients had to score 3 or higher on the TDS to be included in the study. At start of the study, $94.9 \%$ of the patients had a score of 5 , with the remaining $5.1 \%$ having a score of 4 . A success to therapy was defined as a 2-point improvement on the TDS (see Methods). The Wilcoxon signed ranks test was used to analyze changes in TDS over time. Compared with baseline, a significant decrease was observed at BoNT-8 $(\mathrm{Z}=$ $-4.8 ; P=.000,1$-tailed $)$ and BoNT-24 $(\mathrm{Z}=-4.1 ; P=$ $.000,1$-tailed). The TDS is an ordinal scale; as such, the outcome can provide only supportive evidence. To confirm the outcome, the TDS should show changes in the same direction as the VAS (Fig 3). The curve shows that at baseline, all patients were severely drooling (mean: 4.9). Drooling diminished after BoNT injections. At BoNT-8, the mean score decreased to 3, indicating occasional drooling. At BoNT-24, the mean TDS score equaled 3.8.

The TDS was also used to define success to therapy (see Methods). An analysis of frequency of scores on the TDS was made to provide insight into parents' recognition of the BoNT therapy effect. Eight weeks after the BoNT injections, TDS scores were as follows: $1,15.4 \% ; 2,28.2 \%$; and $3,17.9 \%$. This indicates that $61.5 \%$ of the patients were good responders according to our definition; $15.4 \%$ of the children

TABLE 3. Mean Differences Between Baseline and Follow-up Measurements

\begin{tabular}{lccccc}
\hline \multirow{2}{*}{ Pairs of Observation } & \multicolumn{2}{c}{ DQ } & & \multicolumn{2}{c}{ VAS } \\
\cline { 2 - 3 } \cline { 5 - 6 } & Difference (SD) & Significance* & & Difference (SD) & Significance* $^{*}$ \\
\hline $\mathrm{Bl}$ /scopolamine & $17.7(21.2)$ & .000 & & $-34.3(30.9)$ & .000 \\
$\mathrm{Bl} /$ BoNT-2 & $21.7(18.3)$ & .000 & & & \\
$\mathrm{Bl} /$ BoNT-4 & $16.1(18.9)$ & .000 & & $-30.1(22.8)$ & .000 \\
$\mathrm{Bl} /$ BoNT-8 & $20.0(20.5)$ & .000 & & $-22.1(23.9)$ & .000 \\
$\mathrm{Bl} /$ BoNT-16 & $15.5(19.1)$ & .000 & & $-20.5(24.9)$ & .000 \\
$\mathrm{Bl}$ /BoNT-24 & $15.7(16.4)$ & .000 & & $-13.5(25.7)$ & .002 \\
\hline
\end{tabular}

Bl indicates baseline.

* Paired-samples $t$ tests, 1 -sided $P \leq .05$. 
TABLE 4. Mean Differences Between Scopolamine and BoNT Measurements

\begin{tabular}{lrrrrr}
\hline \multirow{2}{*}{ Pairs of Observation } & \multicolumn{2}{c}{ DQ } & & \multicolumn{2}{c}{ VAS } \\
\cline { 2 - 3 } \cline { 5 - 6 } & Difference (SD) & Significance $^{*}$ & & Difference (SD) & Significance* $^{*}$ \\
\hline Sc/BoNT-2 & $4.1(16.5)$ & .131 & & & \\
Sc/BoNT-4 & $-1.6(19.2)$ & .604 & & $4.2(31.3)$ & .405 \\
Sc/BoNT-8 & $2.4(21.7)$ & .502 & & $12.2(30.9)$ & .018 \\
Sc/BoNT-16 & $-2.2(21.8)$ & .528 & & $13.7(32.9)$ & .013 \\
Sc/BoNT-24 & $-2.1(20.2)$ & .529 & & $20.7(39.9)$ & .002 \\
\hline
\end{tabular}

Sc indicates scopolamine.

* With paired-samples $t$ tests, 1 -sided $P \leq .05$.

Fig 2. VAS: mean in time.
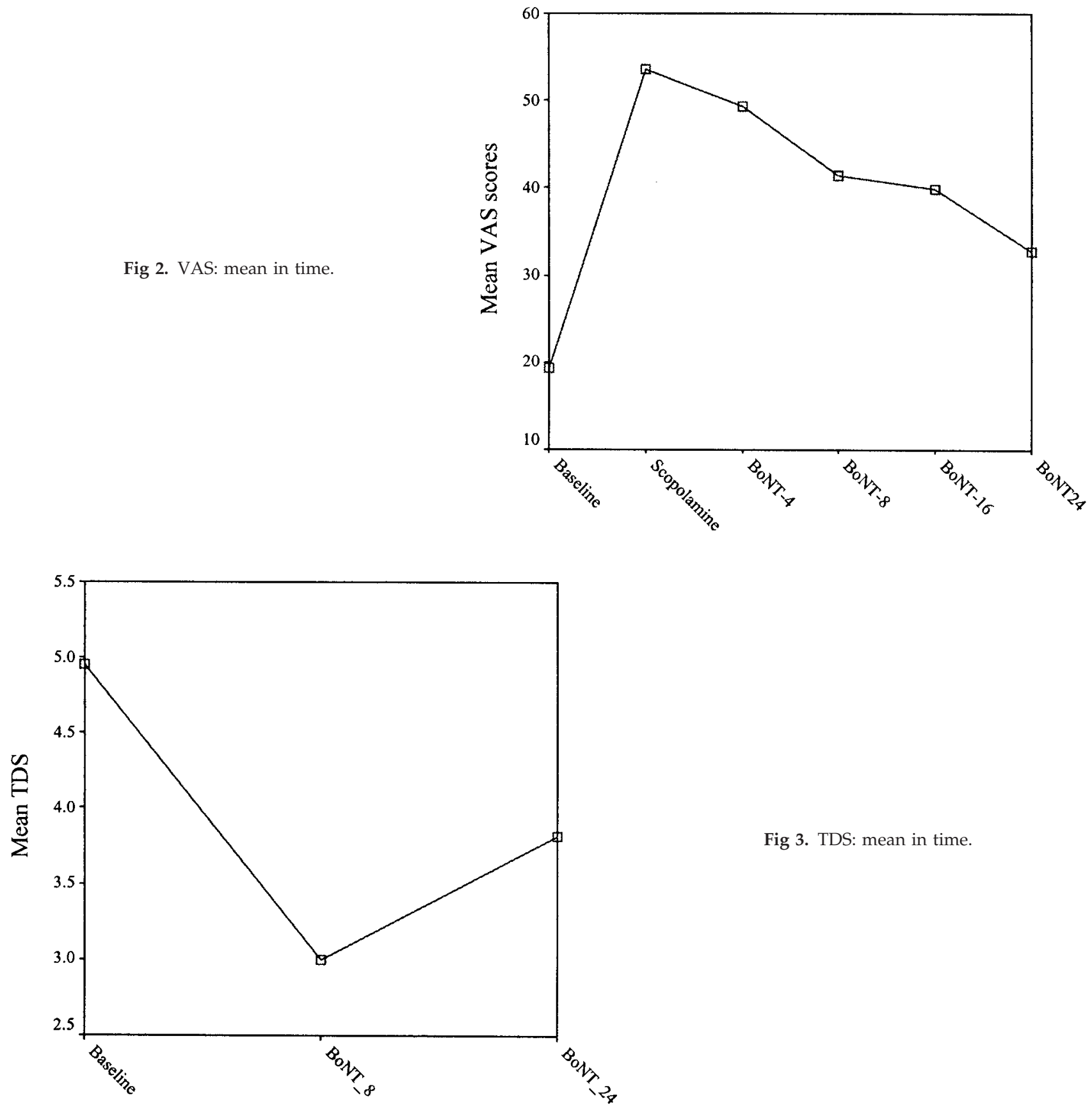

Fig 3. TDS: mean in time.

changed only slightly (score: 4). Twenty-three percent of the cases, representing 9 patients, did not show improvement compared with their initial score. Eight $(20.5 \%)$ patients remained at score 5, and $1(2.5 \%)$ patient remained at score 4 . None of the parents reported an increase in drooling severity as a result of therapy.

None of the patients showed improvement between BoNT-8 and BoNT-24. TDS frequency scores at BoNT-24 were as follows: 1, 5.1\%; 2, 15.4\%; 3, 
$15.4 \% ; 4,20.5 \% ; 5,43.6 \%$. At 24 weeks after BoNT injections, $35.9 \%$ of the parents still reported a good effect, whereas $43.6 \%$ of the children had returned to baseline value, among them the $20.5 \%$ who did not react to therapy at all.

\section{Side Effects}

Parents scored the extent of side effects on a 4-point scale $(0=$ no side effect; $1=$ mild, not every day/occurring sometimes; $2=$ moderate, present every day; 3 = severe side effect, constantly present). Side effects as a result of scopolamine became apparent within the first 3 days of administration, and several patients complained of $>1$ symptom. Adverse effects during application of scopolamine were reported in $82.2 \%$ of cases. Five $(11.1 \%)$ patients had mild, 14 (31.1\%) patients had moderate, and 18 (40\%) patients had severe side effects.

Most often reported adverse effects were xerostomia $(66.7 \%)$, restlessness $(35.6 \%)$, somnolence $(35.6 \%)$, blurred vision because of pupillary dilation, and confusion (20\%). Four of the 6 dropouts from the study had to end their participation because of adverse effects to scopolamine. In all 4 cases, restlessness, apparent in their movement performance, and confusion were the main reasons to terminate scopolamine use. After BoNT injections, incidental side effects were reported. Two (5.1\%) patients had a transient flu-like syndrome that lasted for $<2$ days. Another 3 patients complained of mild difficulty with swallowing.

\section{DISCUSSION}

This is the first controlled, clinical trial to evaluate the treatment of drooling in children with $\mathrm{CP}$ by comparing the efficacy of 2 different anticholinergic agents: bilateral single-dose BoNT injections into the submandibular glands and transdermal scopolamine. A positive clinical effect from intraglandular BoNT injections as well as scopolamine application was found. Scopolamine was expected to have a greater effect on drooling because of its systemic availability, influencing the submandibular glands, the sublingual glands, and the parotid glands apart form all minor glands within the mucosa of the oral cavity. As chosen during this trial, BoNT is injected only focally into the submandibular gland. Nevertheless, the short-term effect on drooling by intraglandular BoNT injections was of the same magnitude as that of scopolamine. This outcome is compatible with the concept that the submandibular glands produce a large part of resting saliva. In addition, it underlines that BoNT has a strong anticholinergic effect in the target glands.

The outcomes of clinically relevant parameters (DQ and VAS) were in accordance with each other, showing a reduction in drooling after both interventions. The parents' reports about their child's drooling at home (VAS scores) showed significant change. A similar positive effect was demonstrated in the reduction of DQ after BoNT injections up to 24 weeks, the end of the study. Response rates, as analyzed in the DQ data, were high. Of all patients, 53\% responded during scopolamine, $64.1 \%$ responded 2 weeks after BoNT injections, and 53\% responded 8 weeks after injections. After 24 weeks, $48.7 \%$ of the patients still responded to BoNT. Success to therapy was also defined as a 2-point decrease of the TDS as compared with baseline. Eight weeks after BoNT injections, $61.5 \%$ of the patients could be registered as responders according to the TDS approach. At the end of the study (BoNT-24), 35.9\% of the patients were still responding to the BoNT injections.

The outcome of success to therapy as described with the DQ is based on an objective observation expressed on a numeric scale. The result of the TDS is a subjective expression on an ordinal scale. Still, the results of both observations on BoNT are congruent. Comparable outcomes on drooling in BoNT studies have been reported in observational studies. 9,10

Transdermally applied scopolamine and oral anticholinergic agents such as glycopyrrolate and benztropine have been widely investigated in the treatment of drooling. 8,15,27-34 Lewis et $\mathrm{al}^{31}$ randomly assigned patients to a 2-week use of scopolamine patches and placebo patches using a crossover design. Success to therapy was recorded in $80 \%$ of patients. Blasco and Stansbury ${ }^{28}$ performed a prospective open-label study in which $90 \%$ of the patients had reduced drooling in response to glycopyrrolate, based on the subjective reports by parents. Bachrach et $\mathrm{al}^{27}$ investigated the results of glycopyrrolate among 37 patients in a questionnaire-based cohort study. Parents were asked to describe the amount of drooling before medication had begun and while their child was taking the medication, using a 5-point scale. A significant improvement was present in $95 \%$ of cases. Mier et $\mathrm{al}^{32}$ used increasing dosages of glycopyrrolate in a placebo-controlled clinical trial. According to their definition of success to therapy, $81 \%$ of patients responded to the highest dosage. The above-mentioned success rates all exceeded the $53 \%$ responders found in the present study. An explanation for this difference could be that the definitions of response (a $50 \%$ reduction or more of the DQ, in our study) used in the studies are not interchangeable.

Disadvantages of systemic anticholinergic drugs are the many side effects. Severe side effects were observed in $40 \%$ of cases. Symptoms such as xerostomia, restlessness, somnolence, blurred vision, and confusion were apparent in this study, necessitating ending the therapy in $7 \%$ of the participants. In this way, 4 of 6 dropouts were attributed to scopolamine. It was theorized that side effects are probably even underscored in this study population, taking into account the patients' inability to present their complaints clearly. Besides, scopolamine was applied for a relatively short period. Thus, continued use of scopolamine in a dosage large enough to treat severe drooling seems undesirable.

Side effects as a result of anticholinergic therapy are also described in other studies. Lewis et $\mathrm{al}^{31}$ reported two third of his population to have pupillary dilatation, and $\sim 27 \%$ demonstrated pruritus or increased mouthing behavior as a reaction to scopolamine. Applying glycopyrrolate, $32.5 \%$ of the pa- 
tients had adverse effects of which behavioral changes $(13 \%)$ were specified in a percentage. ${ }^{28}$ Mier et $\mathrm{al}^{32}$ reported that adverse effects were common, affecting $69 \%$ of the children who took glycopyrrolate. The identified side effects ranged from $10 \%$ to $23 \%$, comprising behavioral changes, facial flushing, nasal congestion, constipation, vomiting, diarrhea, dry mouth, urinary retention, and blurred vision. Seven of 36 participants withdrew from the study because of side effects. Although side effects seem to be common in relation to the use of anticholinergic drugs, the intensity and the occurrence of central effect may vary, depending on the drug of choice.

BoNT led to temporary complaints about swallowing in 2 cases. This was ascribed to local swelling in 1 patient. In another case, moderate difficulty with swallowing developed after 1 week and remained for 10 days. It was theorized that this might have been the result of diffusion of BoNT into the surrounding muscles. Other authors explicitly noted that no side effect were seen after BoNT injections. ${ }^{23}$

BoNT, when accidentally injected next to the salivary glands, will influence neural activity at the neuromuscular junction as well. To avoid side effects and achieve optimal results, ultrasound guidance is strongly recommended.5,23,35,36 Although easy to perform, BoNT injections are to some extent invasive. General anesthesia could be regarded as a disadvantage. In this respect, it must be emphasized that other authors performed the procedure without anesthesia. ${ }^{23}$

As chosen in our protocol, only the submandibular glands were treated. However, it must be realized that there may exist indications to treat the parotids as well, for example, excessive drooling during eating and drinking.

To our knowledge, this is the first controlled clinical trial in the treatment of drooling, evaluating the effect of scopolamine application and intraglandular BoNT injections into the submandibular glands. Both treatments significantly reduced drooling compared with baseline. The outcome of both therapies is in the same range and, no significant differences were found between DQ measurements during scopolamine and BoNT. A disadvantage in the treatment with scopolamine is the high percentage of observed adverse reactions, whereas BoNT injections need general anesthesia in children. The BoNT effect is temporary, although longer duration might be expected especially after recurrent treatment because of supposed hypotrophy of the glands after long denervation. Referring to the demands about the diagnosis and TDS, as stated in the inclusion criteria, the generalizability of this study is limited to comparable groups of patients. Additional research is needed to investigate BoNT therapy in other groups and to compare it with other interventions to treat drooling, such as surgery. Considering the social burden to the affected children, it is relevant to develop clinical guidelines to distinguish types and age groups of drooling patients to optimize the treatment modalities that are specifically effective.

In conclusion, during scopolamine application and after intraglandular BoNT injections, a clinically rel- evant reduction of drooling was achieved in children with $\mathrm{CP}$, demonstrating maximum effect 2 to 8 weeks after injections. Analysis of the DQ demonstrated a response rate to scopolamine of $53 \%$ and of nearly $50 \%$ until 24 weeks after BoNT injections, the actual duration of this study. Additional research is warranted to optimize selection of patients in an effort to maximize the therapeutic effect.

\section{ACKNOWLEDGMENTS}

This work was supported by a grant from the Johanna KinderFonds (Arnhem, Netherlands), a fundraising consortium in the field of child rehabilitation.

We express our gratitude to Frank Joosten, MD, PhD, and Lia van Die, MD, radiologists, and Ricky van der Heijden, trial monitor, for essential professional contributions to the study.

\section{REFERENCES}

1. Ekedahl C. Surgical treatment of drooling. Acta Otolaryngol. 1974;77: 215-220

2. Crysdale WS. Management options for the drooling patient. Ear Nose Throat J 1989;68:820, 825-826, 829-830

3. Harris SR, Purdy AH. Drooling and its management in cerebral palsy. Dev Med Child Neurol. 1987;29:807-811

4. Hussein I, Kershaw AE, Tahmassebi JF, Fayle SA. The management of drooling in children and patients with mental and physical disabilities: a literature review. Int J Paediatr Dent. 1998;8:3-11

5. Ellies M, Laskawi R, Rohrbach-Volland S, Arglebe C, Beuche W. Botulinum toxin to reduce saliva flow: selected indications for ultrasoundguided toxin application into salivary glands. Laryngoscope. 2002;112: $82-86$

6. Jongerius PH, Rotteveel JJ, van den HF, Joosten F, van Hulst K, Gabreels FJ. Botulinum toxin A: a new option for treatment of drooling in children with cerebral palsy. Presentation of a case series. Eur J Pediatr. 2001;160:509-512

7. Rapp D. Drool control: long-term follow-up. Dev Med Child Neurol. 1980;22:448-453

8. Reddihough D, Johnson H, Staples M, Hudson I, Exarchos H. Use of benzhexol hydrochloride to control drooling of children with cerebral palsy. Dev Med Child Neurol. 1990;32:985-989

9. Bothwell JE, Clarke K, Dooley JM, et al. Botulinum toxin A as a treatment for excessive drooling in children. Pediatr Neurol. 2002;27:18-22

10. Suskind DL, Tilton A. Clinical study of botulinum-A toxin in the treatment of sialorrhea in children with cerebral palsy. Laryngoscope. 2002; $112: 73-81$

11. Wong V, Sun JG, Wong W. Traditional Chinese medicine (tongue acupuncture) in children with drooling problems. Pediatr Neurol. 2001; 25:47-54

12. Heine RG, Catto-Smith AG, Reddihough DS. Effect of antireflux medication on salivary drooling in children with cerebral palsy. Dev Med Child Neurol. 1996;38:1030-1036

13. Webb K, Reddihough DS, Johnson H, Bennett CS, Byrt T. Long-term outcome of saliva-control surgery. Dev Med Child Neurol. 1995;37: 755-762

14. Johnson H. An Exploratory Study on Drooling Using a Frequency Method of Measuring in a Naturalistic Setting (Thesis/Dissertation). Melbourne, Australia: La Trobe University; 1990

15. Camp-Bruno JA, Winsberg BG, Green-Parsons AR, Abrams JP. Efficacy of benztropine therapy for drooling. Dev Med Child Neurol. 1989;31: 309-319

16. Crossner CG. Salivary flow rate in children and adolescents. Swed Dent J. $1984 ; 8: 271-276$

17. Heintze U, Birkhed D, Bjorn H. Secretion rate and buffer effect of resting and stimulated whole saliva as a function of age and sex. Swed Dent J. $1983 ; 7: 227-238$

18. Kavanagh DA, O'Mullane DM, Smeeton N. Variation of salivary flow rate in adolescents. Arch Oral Biol. 1998;43:347-352

19. Le Bell Y, Soderling E, Karjalainen S. Effect of repeated sampling and prestimulation on saliva buffer capacity and flow rate values in children. Scand J Dent Res. 1991;99:505-509

20. Lenander-Lumikari M, Puhakka T, Makela MJ, Vilja P, Ruuskanen O, Tenovuo J. Effects of the common cold and intranasal fluticasone propionate treatment on mucosal host defense assessed by human saliva. Oral Surg Oral Med Oral Pathol Oral Radiol Endod. 1999;87:695-699

21. Moret S, Coudert JL, Bejat C, Robin O, Lissac M. The influence of basal 
anxiety on unstimulated parotid and submandibular saliva. Arch Oral Biol. 1993;38:751-754

22. Burton MJ. The surgical management of drooling. Dev Med Child Neurol. 1991;33:1110-1116

23. Ellies M, Laskawi R, Rohrbach-Volland S, Arglebe C. Up-to-date report of botulinum toxin therapy in patients with drooling caused by different etiologies. J Oral Maxillofac Surg. 2003;61:454-457

24. Naumann M, Lowe NJ. Botulinum toxin type A in treatment of bilateral primary axillary hyperhidrosis: randomised, parallel group, double blind, placebo controlled trial. BMJ. 2001;323:596-599

25. Naumann M, Lowe NJ, Kumar CR, Hamm H. Botulinum toxin type a is a safe and effective treatment for axillary hyperhidrosis over 16 months: a prospective study. Arch Dermatol. 2003;139:731-736

26. Blasco PA, Allaire JH. Drooling in the developmentally disabled: management practices and recommendations. Consortium on Drooling. Dev Med Child Neurol. 1992;34:849-862

27. Bachrach SJ, Walter RS, Trzcinski K. Use of glycopyrrolate and other anticholinergic medications for sialorrhea in children with cerebral palsy. Clin Pediatr (Phila). 1998;37:485-490

28. Blasco PA, Stansbury JC. Glycopyrrolate treatment of chronic drooling. Arch Pediatr Adolesc Med. 1996;150:932-935

29. Hyson HC, Johnson AM, Jog MS. Sublingual atropine for sialorrhea secondary to parkinsonism: a pilot study. Mov Disord. 2002;17:1318-1320
30. Jongerius PH, van Tiel P, van Limbeek J, Gabreels FJ, Rotteveel JJ. A systematic review for evidence of efficacy of anticholinergic drugs to treat drooling. Arch Dis Child. 2003;88:911-914

31. Lewis DW, Fontana C, Mehallick LK, Everett Y. Transdermal scopolamine for reduction of drooling in developmentally delayed children. Dev Med Child Neurol. 1994;36:484-486

32. Mier RJ, Bachrach SJ, Lakin RC, Barker T, Childs J, Moran M. Treatment of sialorrhea with glycopyrrolate: a double-blind, dose-ranging study. Arch Pediatr Adolesc Med. 2000;154:1214-1218

33. Neverlien PO, Sorumshagen L, Eriksen T, Grinna T, Kvalshaugen H, Lind AB. Glycopyrrolate treatment of drooling in an adult male patient with cerebral palsy. Clin Exp Pharmacol Physiol. 2000;27:320-322

34. Siegel LK, Klingbeil MA. Control of drooling with transdermal scopolamine in a child with cerebral palsy. Dev Med Child Neurol. 1991;33: 1013-1014

35. Jongerius PH, Joosten F, Hoogen FJ, Gabreels FJ, Rotteveel JJ. The treatment of drooling by ultrasound-guided intraglandular injections of botulinum toxin type A into the salivary glands. Laryngoscope. 2003;113: 107-111

36. Porta M, Gamba M, Bertacchi G, Vaj P. Treatment of sialorrhoea with ultrasound guided botulinum toxin type A injection in patients with neurological disorders. J Neurol Neurosurg Psychiatry. 2001;70:538-540

\section{BLAME LIFESTYLE FOR MYOPIA EPIDEMIC?}

"Contrary to popular belief, people in East Asia are no more genetically susceptible to short-sightedness than any other population group, according to researchers who have analyzed the past studies of the problem. The epidemics of myopia in countries such as Singapore and Japan are due solely to changes in lifestyle, they say, and similar levels could soon be seen in many western countries as lifestyles there continue to change. 'As kids spend more time indoors on computers or watching telly, we are going to become just as myopic,' says Ian Morgan of the Australian National University in Canberra. Myopia is on the increase in most places, but in countries such as Singapore it has reached extraordinary levels. There, 80 percent of 18-year-old male army recruits are myopic, up from 25 percent just 30 years ago.... Another study found myopia rates of 80 percent in 14- to 18 -year-old boys studying in schools in Israel that emphasize reading religious texts. The rate for boys in state schools was just 30 per cent.... In Sweden, for instance, 50 percent of children aged 12 now have myopia. It is expected that when these children reach 18 the rate will be more than 70 percent."

Noted by JFL, MD 


\section{Effect of Botulinum Toxin in the Treatment of Drooling: A Controlled Clinical Trial}

Peter H. Jongerius, Frank J.A. van den Hoogen, Jacques van Limbeek, Fons J.

Gabreëls, Karen van Hulst and Jan J. Rotteveel

Pediatrics 2004;114;620

DOI: 10.1542/peds. 2003-1104-L

\section{Updated Information \& Services}

\section{References}

Citations

Permissions \& Licensing

Reprints including high resolution figures, can be found at: http://pediatrics.aappublications.org/content/114/3/620.full.ht $\mathrm{ml}$

This article cites 35 articles, 4 of which can be accessed free at:

http://pediatrics.aappublications.org/content/114/3/620.full.ht ml\#ref-list-1

This article has been cited by 11 HighWire-hosted articles: http://pediatrics.aappublications.org/content/114/3/620.full.ht ml\#related-urls

Information about reproducing this article in parts (figures, tables) or in its entirety can be found online at:

http://pediatrics.aappublications.org/site/misc/Permissions.xht $\mathrm{ml}$

Information about ordering reprints can be found online: http://pediatrics.aappublications.org/site/misc/reprints.xhtml

PEDIATRICS is the official journal of the American Academy of Pediatrics. A monthly publication, it has been published continuously since 1948. PEDIATRICS is owned, published, and trademarked by the American Academy of Pediatrics, 141 Northwest Point Boulevard, Elk Grove Village, Illinois, 60007. Copyright ( 2004 by the American Academy of Pediatrics. All rights reserved. Print ISSN: 0031-4005. Online ISSN: 1098-4275.

\section{American Academy of Pediatrics}

DEDICATED TO THE HEALTH OF ALL CHILDREN ${ }^{m}$ 